\title{
Three-Dimensional Analysis of Variants of Division of the Renal Artery and Its Branches
}

\author{
Vezirkhanov A.Z.* Dokayeva T.S. Kafarov E.S.
}

\author{
Department of Normal and Topographic Anatomy and Operative Surgery, Federal State budgetary educational, \\ Chechen state university, Grozny, Russia \\ *Corresponding author. Email: selim2005@mail.ru
}

\begin{abstract}
The aim of the study was to conduct a three-dimensional analysis of some variants of a renal artery' division and its major vessels. We performed 124 corrosive preparations of a human kidney arterial system, followed by 3D scanning to obtain digital models. In a three-dimensional projection, the variants, and types of vascular branching of the arterial system of the human kidney were determined with the number of branches of the 1st, 2nd, and 3rd orders. The morphometric analysis data were processed by the methods of variation statistics. It was found that $54.2 \%$ of renal artery in the hilum is divided into 2 branches: ventral and dorsal. In $15.5 \%$ of cases, the renal artery is divided in the horizontal plane into the upper pole and lower pole branches. In $12.9 \%$ of cases, it is divided into ventral, dorsal and upper pole branches; in $9.4 \%$ of cases, there is a division of the renal artery and into the ventral, dorsal and lower pole branches; in $5.1 \%$ of cases, it divides into two ventral and one dorsal branches in the frontal plane; in $2.5 \%$ of cases, the renal artery is divided into the upper pole, central and lower pole branches.
\end{abstract}

Keywords: kidney, arteries, options

\section{INTRODUCTION}

Works of many authors highlight various options for the division of the renal artery [1-6, 8-12]. So, one of the first works on this issue was the work of Serov V.V. (1959) [10]. According to the author, renal artery is divided into two trunks in $78 \%$ of cases. In $15 \%$ of cases, it is divided into three trunks and in $7 \%$ of cases into four. When the renal artery is divided into two trunks, which is in $84.6 \%$ of cases, these were the anterior and posterior lochanochnic branches, and in $15.4 \%$ of cases, there were the upper and lower polar branches encountered. The author states that when dividing the renal artery into 3 trunks, most often these were the anterior lochanochnic, retilochanteric and upper polar arteries. When dividing the renal artery into four trunks, most often these were anterior-lochanochnic, retilochanteric, upper polar and lower polar arteries. As for the types of branching of these vessels, according to Serov V.V. (1959), the main type of branching of the anterior lochanteric artery was found in $13.9 \%$ of cases, in $83.5 \%$ of cases a loose type of branching of vessels was found, and in $2.6 \%$ of cases it was a transitional type of branching of these vessels was [10].

The author found that the main type of branching of the retilochanteric artery is the trunk artery, which was found in $62.4 \%$ of cases. The loose type of branching was found in $35.3 \%$ of cases, and in $2.3 \%$ of cases - the mixed type. In cases of division of the renal artery into upper and lower branches, in $64.5 \%$ of cases, the upper pole branch had a loose type of vascular branching, in $32.3 \%$ of cases it had a main type, and in $3.2 \%$ of cases it was mixed. Khomenko V.F. (1962) also established the division of the main trunk of the renal artery into two branches in $90.3 \%$ of cases. The author found that in $8.7 \%$ of cases the renal artery is divided into three branches, in $1 \%$ of cases the renal artery is divided into 4 branches [11].

Research performed by Serov V.V. (1959), Eremeeva S. G. (1962), Khomenko V.F., (1962) almost coincide with the data of Kazartseva M.S. (1969), who discovered the division of the renal artery into two branches in $77.9 \%$ of cases $[2,3,10,11]$. At the same time, the renal artery was divided into the anterior and posterior lohse branches in $55.3 \%$ of cases; on the upper and lower pole branches in $22.6 \%$ of cases. Division of the renal artery into three branches was found in $20.7 \%$ of cases.

The author found that in $14.7 \%$ of cases, the renal artery was divided into the anterior lohse, retilochanteric and lower polar arteries. In $6 \%$ of cases, the renal artery is divided into the anterior lohse, retilochanteric and upper pole branches. Four branches of the renal artery were identified by the author in $1.4 \%$ of cases [3].

According to Burykh M. P. (1968), revealed the division of the renal artery into five branches. However, the author states that he did not reveal significant differences in the number of branches that are primarily separated from the right and left renal arteries.

The author also claims that there is not even a noticeable difference in the frequency of loose and main types of branching of the renal artery on the right and left side. At the hilum of the kidney, each primary branch of the renal artery gives off segmental branches of the second order, but the author does not give the frequency of occurrence [1].

In addition, the issues of architectonics of the renal arteries were studied by Olofinsky L.A. (1970), 
Rubinov Y.L. (1972) [8, 9]. According to lofinsky L.A. (1970) found that in $81.2 \%$ of observations, the renal artery is divided into two branches, namely in front - and retilochanteric one. The author found that in $49.3 \%$ of cases, the anterior lohse branch had a loose type of branching, and the posterior lokhanum branch had a main branch. However, in $26.7 \%$ of cases, both branches of the renal artery had the main type of vascular branching, and in $24 \%$ of cases - the loose one [8]. The data of the above authors correspond to the studies of Kafarova E.S. (2008), who discovered the division of the renal artery into two branches in $85 \%$ of cases [6]. At the same time, the author found that $72.5 \%$ the renal artery is divided into anterior and posterior artery, and in $12.5 \%$ of cases into the superior and inferior polar arteries. In $15 \%$ of cases, the renal artery is divided into three vessels: the upper pole, the anterior lohse, and the retilochanteric arteries, while the latter two arteries always had the main branching type [6].

However, despite the fact that today more and more attention is being paid to the study of the renal arterial bed, there is still no single point of view on a number of issues: options for the divergence of the renal arteries, their number and location in the renal pedicle. The available literature provides conflicting information about the number and variants of vessels, which became the purpose of the study.

\section{METHODS AND MATERIALS}

116 corrosive preparations of human renal arterial vessels were manufactured for the study. Corrosion preparations were subjected to 3D digital scanning. After scanning, computer processing was carried out in special programs "Mimics-8.1". The computer program "Mimics-8.1" was used to determine the projection of arterial vessels of a kidney in relation to the frontal, sagittal and horizontal planes. The program determined types of intraorgan branching of branches of the renal artery and the number of vessels of different orders depending on the types of branching of the arteries. The article studied stereoprojection of variants, types of vascular branching of the arterial system of a human kidney, identification of the number of branches of the 1st, 2nd, and 3rd orders.

Statistical processing included the calculation of the main indicators of random variables distribution: median, mean, quartiles, confidence interval, minimum and maximum values, variance, standard deviation, mean error, median error. We used a licensed package of applied statistical programs MedStat in accordance with the recommendations [7].

\section{RESULTS}

We carried out a three-dimensional anatomical analysis of division variants of the right and left main renal arteries in the renal hilum in the sagittal plane, tangent to the medial border of kidneys in both sexes. It was revealed that in men, the main right renal artery in $32.7 \%$ of cases is divided at the hilum of a kidney, that is, lateral in the sagittal plane, tangent to the medial edge of a kidney (intrarenal variant), in $67.3 \%$ of cases - at a distance from the hilus of a kidney, that is, medial to the sagittal plane, tangent to the medial border of kidneys (extrarenal option). On the left, the main renal artery is divided in $38.4 \%$ of cases at the hilum of a kidney, that is, laterally, in the sagittal plane, tangent to the medial edge of the kidneys (intrarenally) (Fig. 4), and in $61.6 \%$ of cases, medially from the sagittal plane, tangent to the medial border of the kidneys - extrarenally. In women, the division of the right main renal artery in $37.2 \%$ of cases occurs at the hilum of a kidney, that is, lateral to the sagittal plane, tangent to the medial edge of a kidney - intrarenally, and in $62.7 \%$ of cases, medial to the sagittal plane of the tangent to the medial edge of the kidney - extrarenally. On the left, the main renal artery in $31.7 \%$ of cases is formed at the hilum of a kidney, that is, lateral from the sagittal plane of the tangent to the medial edge of s kidney - intrarenally, and in $68.3 \%$ of cases, medially from the sagittal plane, tangent to the medial edge of the kidney - extrarenally.

The authors carried out three-dimensional analysis of the variants of division of the main renal artery relative to frontal, horizontal and sagittal planes.

It was revealed that on 81 corrosive preparations of human renal arterial vessels out of 116 in this group, the main renal artery was divided into two branches, which was found in $69.8 \%$ of cases. At the same time, division of the main renal artery in the frontal plane into ventral and dorsal branches was revealed in $54.2 \%$ of cases (63 preparations out of 81). In $15.5 \%$ of cases (18 preparations), the main renal artery, in the horizontal plane, was divided into the superior pole and inferior pole branches. Furthermore, we encountered variants with 35 corrosive preparations of arterial vessels out of 116; the main renal artery was divided into 3 branches, which was revealed in $30.1 \%$ of cases. At the same time, in $12.9 \%$ of cases out of 35 corrosive preparations (15 preparations), the main renal artery in the frontal and horizontal planes, was divided into ventral, dorsal and upper pole branches; in $9.4 \%$ of cases (11 preparations), the main renal artery in frontal and horizontal planes was divided into ventral, dorsal and inferior pole branches; in $5.1 \%$ of cases (6 preparations), the main renal artery was divided into two ventral and one dorsal branches in the frontal plane; in $2.5 \%$ of cases ( 3 preparations), the main renal artery was divided into the upper pole, central, and lower pole branches.

As it was noted, out of 116 corrosive preparations of human renal arterial vessels on 63 preparations, the main renal artery in the frontal plane was divided into ventral and dorsal branches (54.2\% of cases). At the same time, of 63 preparations on 19 preparations, the main renal artery was divided at the hilum of a kidney, that is, lateral to the sagittal plane, tangent to the medial edge of the kidney (intrarenal variant), which accounted for $30.1 \%$ of cases. In $69.8 \%$ of cases (44 preparations), the main renal artery was divided at a distance from the hilum of the 
kidneys, that is, medial to the sagittal plane, tangent to the medial edge of kidneys (extrarenal variant).

1. It is important to pay attention to the group of corrosive preparations of arterial vessels, where the main renal artery was divided into two branches (ventral and dorsal, 44 preparations). This was encountered in $69.8 \%$ of cases relatively far from the hilum of a kidney extrarenal. At the same time, on 24 corrosive preparations, the ventral branch of the main renal artery was divided into $4 \pm 1$ vessels of the 3 rd order and from 4 to 6 vessels of the 4th order, and the dorsal branch was divided into $3 \pm$ 1 and from 4 to 8 vessels of the 4th order diverging from the place of its formation. It was distributed in the ventral and dorsal parts of the kidney parenchyma, which we met in $30.0 \%$ of cases.

On 16 corrosive preparations, the ventral branch of the main renal artery was divided into $3 \pm 1$ vessels of the 3 rd order and from 4 to 7 vessels of the 4 th order, and the dorsal branch was divided into $2 \pm 1$ and from 4 to 6 vessels of the 4th order, which we found $25.3 \%$ of cases. On 4 corrosive preparations, the ventral and dorsal branches of the renal artery were divided into $3 \pm 1$ vessels of the 3 rd order and from 5 to 7 vessels of the 4th order, which we encountered in $6.3 \%$ of cases.

2. As mentioned above, out of 63 corrosive preparations, 19 main renal artery was divided at the hilum of the kidney, lateral to the sagittal plane, tangent to the medial edge of the kidneys (intrarenal variant), which accounted for $30.1 \%$ of cases. In this variant, on 11 corrosive preparations out of 19 , the ventral branch of the main renal artery was divided into $3 \pm 1$ vessels of the 3 rd order and from 4 to 8 vessels of the 4 th order, and the dorsal branch was divided into $2 \pm 1$ and from 6 to 8 vessels of the $2 \mathrm{~d}$ order, which were distributed in the ventral and dorsal parts of the renal parenchyma, which we met in $17.4 \%$ of cases. In 5 corrosive preparations out of 19 , the ventral branch of the main renal artery was divided into $4 \pm 1$ vessels of the 3rd order and from 6 to 8 vessels of the 4th order, and the dorsal branch was divided into $2 \pm 1$ and from 4 to 6 vessels of the 4 th order, that we met $7.9 \%$ of cases.

The main renal artery was divided into a different number of arteries (from 2 to 3 ). The most numerous was the group of corrosive preparations of renal vessels, where the main renal artery was divided into the ventral and dorsal branches relative to the frontal plane, which we found in $54.2 \%$ of cases (63 preparations). At the same time, a pronounced individual variability was revealed in the nature of branching and distribution of arteries of the $3 \mathrm{~d}$ and 4 th orders.

Of 63 preparations, which is in $46.2 \%$ of cases, we found preparations where in the dorsal parts of the renal parenchyma from the dorsal artery with the main branching trunk $5 \pm 1$ vessels of the 3 rd order departed from the dorsal artery. They were distributed in the dorsal parts of the renal parenchyma. In the ventral parts of the kidney, $4 \pm 1$ interlobar arteries of the third order with approximately the same diameter departed from the ventral branch of the main renal artery. Therefore, we can speak of a mixed type of branching for a kidney.
The next group was represented by preparations where the ventral and dorsal arteries had loose branching, which we found in $23.3 \%$ of cases. At the same time, the ventral branch gave off $5 \pm 1$ vessels of the third order, distributed in the ventral parts of the renal parenchyma, and the dorsal $4 \pm 1$ vessels of the third order with approximately the same diameter, branching in the dorsal parts of the kidney.

In $19.4 \%$ of cases, we found preparations where both ventral and dorsal branches of the main renal artery had main trunks, from which $5 \pm 1$ vessels of the 3rd order also departed, branching in the ventral and dorsal parts of the renal parenchyma.

The 4th group was represented by preparations, where the ventral branch of the main renal artery had the main branching type, giving off $5 \pm 1$ vessels of the 3 rd order for the ventral parts of the kidney, and the dorsal loose one, from which $4 \pm 1$ vessels of the 3 rd order departed to the dorsal parts kidneys, which were found in $11.1 \%$ of cases. Studies have shown that the division of the main renal artery most often occurs extrarenally into ventral and dorsal vessels.

\section{CONCLUSION}

Thus, it was revealed that in men the renal artery on the right side of the body is divided in $32.7 \%$ of cases at the hilum of a kidney (intrarenal variant). In $67.3 \%$ of cases, an extrarenal variant of renal artery division was found. On the left of the body, the renal artery is divided in $38.4 \%$ of cases at the hilum of a kidney (intrarenal variant). In $61.6 \%$ of cases, it was an extrarenal variant of renal artery division. In women on the right side of the body, renal artery is divided intrarenally in $37.2 \%$ of cases, and extrarenally in $62.7 \%$ of cases. On the left of the body, the renal artery is divided intrarenally in $31.7 \%$ of cases, and an extrarenal variant of renal artery division was revealed in $68.3 \%$ of cases. It was found that $54.2 \%$ of cases of renal artery in the hilum is divided into 2 branches: ventral and dorsal. In $15.5 \%$ of cases, the renal artery is divided into the upper pole and lower pole branches in the horizontal plane. In $12.9 \%$ of cases, it is divided into ventral, dorsal and upper pole branches; in $9.4 \%$ of cases, there is a division of the renal artery and into the ventral, dorsal and lower pole branches; in $5.1 \%$ of cases, it divides into two ventral and one dorsal branches relative to the frontal plane, and in $2.5 \%$ of cases, the renal artery is divided into the upper pole, central and lower pole branches.

\section{ACKNOWLEDGMENT}

The article was published as part of the implementation of the RFBR grant in accordance with contract 20-315-90008 


\section{REFERENCES}

[1] M.P. Burykh, Nerves and vessels of the kidneys of humans and some animals, Ph.D. Thesis, Kharkov, 1968, $40 \mathrm{p}$.

[2] S.G. Eremeev, Arterial segments of the kidneys, Ph.D. Thesis, Voronezh, 1962, 17 p.

[3] M.S. Kazartsev, Age features of the segmental structure of human kidneys, Ph.D. Thesis, Voronezh, 1969, 23 p.

[4] E.S. Kafarov, Variant anatomy of the renal artery and its branches, Ph.D. Thesis, Volgograd, 2004, 19 p.

[5] E.S. Kafarov, Structural transformations of the venous vessels of the human kidneys in mature, elderly and senile age, Ph.D. Thesis, Ufa, 2014, 45 p.

[6] E.S. Kafarov, F.R. Asfandiyarov, M.N. Trizno, Types of branching of arterial and venous vessels of the kidney, Morphological news 3-4 (2008) 41-42.
[7] Y.E. Lyakh, V.G. Guryanov, V.N. Khomenko et al., Fundamentals of computer biostatistics: analysis of information in biology, medicine and pharmacy using the statistical package MedStat, 2006, 214 p.

[8] L.A. Olofinsky, The surgical significance of the relationship of the vascular architectonics of the kidney with the structure of the calyx-pelvic system and the external shape of the organ, Ph.D. Thesis, Vladivostok, 1970, $15 \mathrm{p}$.

[9] Y.M. Rubinov, Surgical anatomy of the kidneys, Ph.D. Thesis, Ryazan, 1972, 15 p.

[10] V.V. Serov, Segmental structure of the vascular system of the kidney, Urology 3 (1959) 6-12.

[11] V.F. Khomenko, To the surgical anatomy of the arterial vessels of the kidney, Urology 3 (1962) 13-18.

[12] F.J. Sampaio, J.L. Schiavani, L.A. Favorito, Proportional analysis of the kidney arterial segments, Urol. Res. 21(6) (1993) 371-374. 